Reduction of bone cancer pain by CB1 activation and TRPV1 inhibition

Tomoyuki Kawamata, Yukitoshi Niiyama, Jun Yamamoto and Shingo Furuse

Department of Anesthesiology, Sapporo Medical University School of Medicine 
As advances in cancer detection and treatment have increased the life expectancy of cancer patients, more attention to improving patient quality of life is needed. Among the various types of cancer pain, bone cancer pain is often debilitating, difficult to treat, and insufficiently relieved. Recently, a preclinical model of bone cancer pain has been developed [1]. This model has begun to provide insights into the mechanisms by which tumors cause pain and how cancer pain-related sensory information is processed. Here we present new strategies for bone cancer pain based on our data obtained from analyses of a mouse model of bone cancer pain.

1. Murine model of bone cancer pain

The murine model of bone cancer pain is made by means of injection of osteosarcoma cells into the intramedullary space of the femur [1]. Although bone marrow cells fill the intramedullary space in the normal mouse femur, they are largely replaced by tumor cells in the femur at day 14 after osteosarcoma injection (Figure 1). This model features a reproducible pattern and rate of tumor growth within the intramedullary cavity following implantation of sarcoma cells [2]. Figure 2 shows pain-related behavior before and at day 7, day 14 and day 21 after sarcoma or sham injection. The mice with sham injection exhibit no detectable pain-related behavior throughout the observation period. On the other hand, mice with osteosarcoma injection exhibit spontaneous flinches and impaired weight-bearing at day 7. These pain-related behaviors remain unchanged at days 14 and 21 . Mice with sarcoma injection also exhibit impaired limb use during spontaneous ambulation at day 7. At days 14 and 21, more pronounced impairment of limb use is observed.

\section{Transient receptor potential vanilloid subfamily 1 (TRPV1)}

TRPV1 is expressed predominantly in unmyelinated neurons and is activated not only by capsaicin but also by noxious heat, protons, and membrane-derived lipids [3]. Because these stimuli cause pain in vivo, this sensitivity of TRPV1 to multiple types of noxious stimuli might explain the properties of so-called polymodal nociceptors. TRPV1 is essential for selective modalities of pain as 
well as for tissue injury-induced thermal hyperalgesia [4]. Pharmacological studies have also suggested a contribution of TRPV1 to hyperalgesia observed in neuropathic pain and diabetes $[5,6]$. In addition, change in the pattern of expression and up-regulation of TRPV1 in DRG neurons can contribute to abnormal increase in pain transmission. However, the potential role of TRPV1 in bone cancer pain is unknown. Therefore, we examined the involvement of TRPV1 in bone cancer pain.

(1) Analgesic effects of TRPV1 inhibition on bone cancer pain [7]

Systemic administration of a potent TRPV1 antagonist, 5-iodoresiniferatoxin, reduced bone cancer-related pain behaviors without producing any observable side effects in a dose-dependent fashion at day 14 after sarcoma injection.

(2) Altered expression of TRPV1 in DRG neurons in bone cancer pain [7]

TRPV1 level was significantly increased in DRG neurons and peripheral axons ipsilateral to the site of osteosarcoma injection in a transcription-dependent fashion at day 14 after injection. Sarcoma injection increased the number of TRPV1-positive neurons, and a TRPV1-positive profile was observed not only in small neurons but also medium to large neurons in ipsilateral DRGs. The percentage of the ratio of TRPV1-positive neurons was notably increased in neurons positive for NF200 (a marker of neurons with myelinated fibers) and in neurons positive for CGRP (a marker of peptidergic neurons) but not in neurons positive for IB4 (a marker of non-peptidergic neurons).

(3) Increased excitability of TRPV1 [8]

Many chemical mediators released from tumor cells and inflammatory cells can be involved in bone cancer pain. Among the various chemical mediators, endothelin-1 (ET-1) is released by oseteosarcoma cells, and activation of endothelin type A receptor (ETA) by ET-1 generates bone cancer pain. We performed an in vitro experiment on interaction of TRPV1 with ET-1. We found that ET-1 sensitized TRPV1 in an ETA-PKC-dependent manner and that the thermal threshold of TRPV1 
was decreased below normal body temperature.

(4) Rescue of decreased analgesic effect of morphine by a TRPV1 antagonist

The analgesic effect of intrathecal morphine in bone cancer pain is decreased compared to that in non-malignant inflammatory pain. Decreased analgesic effect of morphine in bone cancer pain is associated with down-regulation of the $\mu$ opioid receptor in TRPV1-positive DRG neurons [9]. The combination of morphine and a TRPV1 antagonist, SB366791, was shown to have a potent analgesic effect on bone cancer pain, and a sub-analgesic dose of SB366791 therefore potentiated the reduced analgesic effect of morphine [10]. Thus, the combination of morphine and a TRPV1 antagonist can rescue the decreased analgesic effect of morphine.

Our results show that TRPV1 is an important molecule in the generation of bone cancer pain and a target for treatment of bone cancer pain.

\section{Cannabinoid receptor type I (CB1)}

Recently, there has been increasing interest in the use of cannabinergic system-based drugs for the management of pain. Cannabinoids exert their effects via activation of $\mathrm{CB} 1$ and $\mathrm{CB} 2$, which are coupled to $\mathrm{G}_{\mathrm{i} / \mathrm{o}}$ protein. The role of spinal CB1 in nociceptive transmission has been extensively studied. Activation of spinal CB1 has been shown to inhibit glutamatergic excitatory postsynaptic currents in spinal cord slices of naïve rats [11] and c-fiber-evoked neuronal response of the dorsal horn [12-14]. It has also been shown that spinal CB1 activation reduced several types of pain [15, 16]. However, the role of spinal CB1 in bone cancer pain has not been elucidated. Therefore, we focused on spinal CB1 to develop a novel strategy for the treatment of bone cancer pain.

(1) Analgesic effect of spinal CB1 activation on bone cancer pain [17]

Spinal CB1 activation by the CB1 agonist arachidonyl-2-chloroethylamide reduced bone cancer-related pain behaviors in a dose-dependent manner without inducing catalepsy and motor impairment, which were associated with systemic administration of CB1 agonists. However, spinal 
inhibition of the metabolism of two endogenous cannabinoids, anandamide and 2-arachidonoyl glycerol, and inhibition of spinal CB1 did not affect bone cancer pain. These results suggest that nociceptive input by bone cancer does not activate the spinal endocannabinoid system involving CB1.

(2) Expression of CB1 in the spinal cord in a murine model of bone cancer pain [25]

In the dorsal horn of the spinal cord, CB1 was mainly expressed in specific laminae including the superficial dorsal horn, deep layer of the dorsal horn, dorsolateral funiculus and around the central canal. In the superficial dorsal horn, which plays an important role in the processing of nociceptive transmission from the periphery to the central nervous system, CB1 was expressed in lamina I and lamina II outer and the ventral part of lamina II inner but not in the dorsal part of lamina II inner. Dorsal root rhizotomy did not alter expression of CB1 in the dorsal horn, and CB1 was not colocalized with any neuronal makers of primary afferents including CGRP, IB4 and cholera toxin $\beta$ subunit. In addition, CB1 was expressed within the axon terminals of the spinal interneurons rather than in the dendritic processes. In contrast to MOR expression, CB1 expression in the superficial dorsal horn ipsilateral to the site of implantation of sarcoma cells was preserved, compared to that contralateral to the site of implantation.

The results of our study demonstrate that spinal CB1 activation by an exogenously administered CB1 agonist reduced bone cancer-related pain behaviors, including behaviors related to spontaneous pain and movement-evoked pain. Presynaptic inhibition of spinal neurons may contribute to spinal CB1 activation-induced analgesia.

4. Mechanism and possible therapeutic strategies of bone cancer pain

The possible mechanism of bone cancer pain based on our data is shown in the figure 2 . TRPV1 is an important molecule in the generation of bone cancer pain and a target for treatment of bone cancer pain. Expression of CB1, but not that of MOR, is preserved in the dorsal horn of the 
spinal cord under a bone cancer pain condition. Therefore, spinal CB1 activation may be effective for the treatment of bone cancer pain, compared to MOR activation. The reduced analgesic effect of morphine can be rescued by its combination with a TRPV1 antagonist. In addition to our findings, other researchers have shown that bradykinin, prostaglandin E2, ET-1, nerve growth factor and osteoclasts are important molecules in the generation of bone cancer pain. It is expected that these findings will lead to mechanism-based treatment of bone cancer pain, resulting in improvement in QOL of cancer patients. 


\section{References}

1. Schwei MJ, Honore P, Rogers SD, Salak-Johnson JL, Finke MP, Ramnaraine ML, Clohisy DR, Mantyh PW. Neurochemical and cellular reorganization of the spinal cord in a murine model of bone cancer pain. J Neurosci. 1999; 19:10886-97.

2. Peters CM, Ghilardi JR, Keyser CP, Kubota K, Lindsay TH, Luger NM, Mach DB, Schwei MJ, Sevcik MA, Mantyh PW. Tumor-induced injury of primary afferent sensory nerve fibers in bone cancer pain. Exp Neurol. 2005; 193:85-100.

3. Tominaga M, Caterina MJ, Malmberg AB, Rosen TA, Gilbert H, Skinner K, Raumann BE, Basbaum AI, Julius D. The cloned capsaicin receptor integrates multiple pain-producing stimuli. Neuron. 1998; 21:531-43.

4. Caterina MJ, Leffler A, Malmberg AB, Martin WJ, Trafton J, Petersen-Zeitz KR, Koltzenburg M, Basbaum AI, Julius D. Impaired nociception and pain sensation in mice lacking the capsaicin receptor. Science. 2000; 288:306-13.

5. Pomonis JD, Harrison JE, Mark L, Bristol DR, Valenzano KJ, Walker K. N-(4-Tertiarybutylphenyl)-4-(3-cholorphyridin-2-yl)tetrahydropyrazine-1(2H)-carbox-amide (BCTC), a novel, orally effective vanilloid receptor 1 antagonist with analgesic properties: II. in vivo characterization in rat models of inflammatory and neuropathic pain. J Pharmacol Exp Ther. 2003; 306:387-93.

6. Rashid $\mathrm{MH}$, Inoue $\mathrm{M}$, Bakoshi $\mathrm{S}$, Ueda $\mathrm{H}$. Increased expression of vanilloid receptor 1 on myelinated primary afferent neurons contributes to the antihyperalgesic effect of capsaicin cream in diabetic neuropathic pain in mice. J Pharmacol Exp Ther. 2003; 306:709-17.

7. Niiyama Y, Kawamata T, Yamamoto J, Omote K, Namiki A. Bone cancer increases transient receptor potential vanilloid subfamily 1 expression within distinct subpopulations of dorsal root ganglion neurons. Neuroscience. 2007; 148:560-72. 
8. Kawamata T, Ji W, Yamamoto J, Niiyama Y, Furuse S, Namiki A. Contribution of transient receptor potential vanilloid subfamily 1 to endothelin-1-induced thermal hyperalgesia. Neuroscience. 2008; 154:1067-76.

9. Yamamoto J, Kawamata T, Niiyama Y, Omote K, Namiki A. Down-regulation of mu opioid receptor expression within distinct subpopulations of dorsal root ganglion neurons in a murine model of bone cancer pain. Neuroscience. $2008 ; 151: 65-72$.

10. Niiyama Y, Kawamata T, Yamamoto J, Furuse S, Omote K, Namiki A. SB366791, a TRPV1 antagonist, potentiates analgesic effects of systemic morphine on bone cancer pain. Brit $\mathrm{J}$ Anaesth. 2009; 102: 251-8.

11. Morisset V, Urban L: Cannabinoid-induced presynaptic inhibition of glutamatergic EPSCs in substantia gelatinosa neurons of the rat spinal cord. J Neurophysiol 2001; 86: 40-8.

12. Kelly S, Chapman V. Selective cannabinoid CB1 receptor activation inhibits spinal nociceptive transmission in vivo. J Neurophysiol. 2001; 86: 3061-4.

13. Sagar DR, Kelly S, Millns PJ, O'Shaughnessey CT, Kendall DA, Chapman V. Inhibitory effects of $\mathrm{CB} 1$ and $\mathrm{CB} 2$ receptor agonists on responses of DRG neurons and dorsal horn neurons in neuropathic rats. Eur J Neurosci. 2005; 22: 371-9.

14. Drew LJ, Harris J, Millns PJ, Kendall DA, Chapman V. Activation of spinal cannabinoid 1 receptors inhibits C-fibre driven hyperexcitable neuronal responses and increases [35S]GTPgammaS binding in the dorsal horn of the spinal cord of noninflamed and inflamed rats. Eur J Neurosci. 2000; 12: 2079-86.

15. Rahn EJ, Makriyannis A, Hohmann AG. Activation of cannabinoid CB1 and CB2 receptors suppresses neuropathic nociception evoked by the chemotherapeutic agent vincristine in rats. Br J Pharmacol. 2007; 152: 765-77.

16. Agarwal N, Pacher P, Tegeder I, Amaya F, Constantin CE, Brenner GJ, Rubino T, Michalski 
CW, Marsicano G, Monory K, Mackie K, Marian C, Batkai S, Parolaro D, Fischer MJ, Reeh P, Kunos G, Kress M, Lutz B, Woolf CJ, Kuner R. Cannabinoids mediate analgesia largely via peripheral type 1 cannabinoid receptors in nociceptors. Nat Neurosci. 2007; 10: 870-9.

17. Furuse S, Kawamata T, Yamamoto J, Niiyama Y, Omote K, Watanabe M, Namiki A. Reduction of bone cancer pain by activation of spinal cannabinoid receptor 1 and its expression in the superficial dorsal horn of the spinal cord in a murine model of bone cancer pain. Anesthesiology. $2009 ; 111: 173-86$. 


\section{Figure legends}

Figure 1.

A, Hematoxylin-eosin staining of normal femur (A-1) and sarcoma-bearing femur (14 days after sarcoma injection) (A-2). Note the replacement of the darkly stained marrow cells with the more lightly stained sarcoma cells in the sarcoma-bearing femur. B, Time course of bone cancer pain-related behaviors. All behaviors were analyzed before (at day 0), at day 7, 14, and 21 after sarcoma or sham injection. $B-1$, the number of spontaneous flinches during a 2 min observation period. $B-2$, the limb use score during spontaneous ambulation. $B$-3, weight bearing score during spontaneous standing. Sarcoma, mice with sarcoma injection; normal, untreated mice; sham, mice with sham injection.

\section{Figure 2.}

Possible mechanism of bone cancer pain. Under a bone cancer condition, TRPV1 is up-regulated in the peripheral neurons and sensitized. On the other hand, MOR is down-regulated in the peripheral neurons. CB1 is located in the axon terminals of excitatory spinal interneuron, and its expression is preserved under a bone cancer condition. 
A
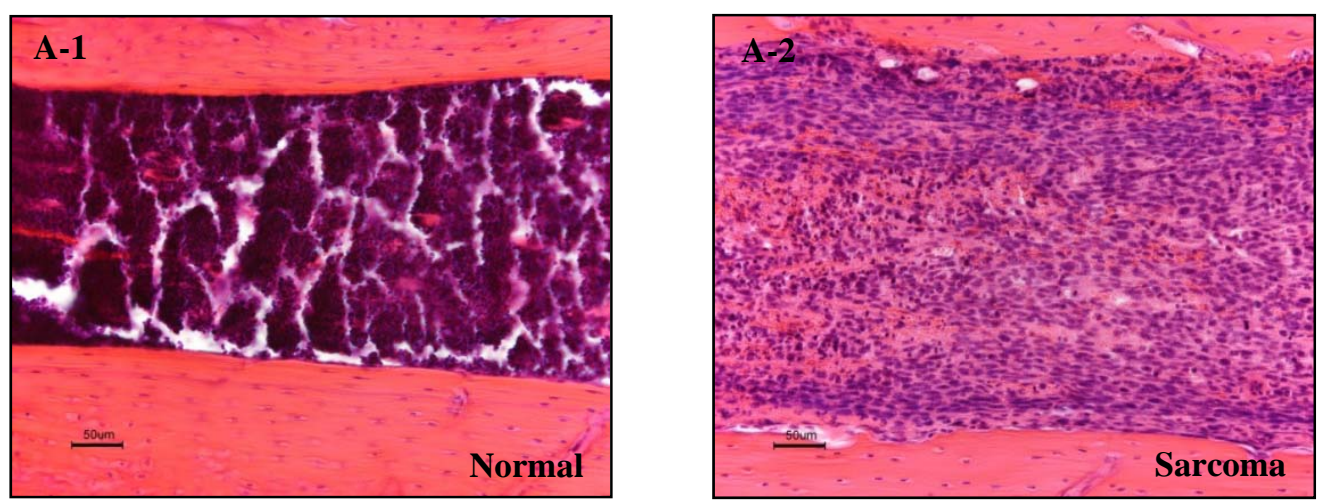

B

\section{B-1. Spontaneous flinches}

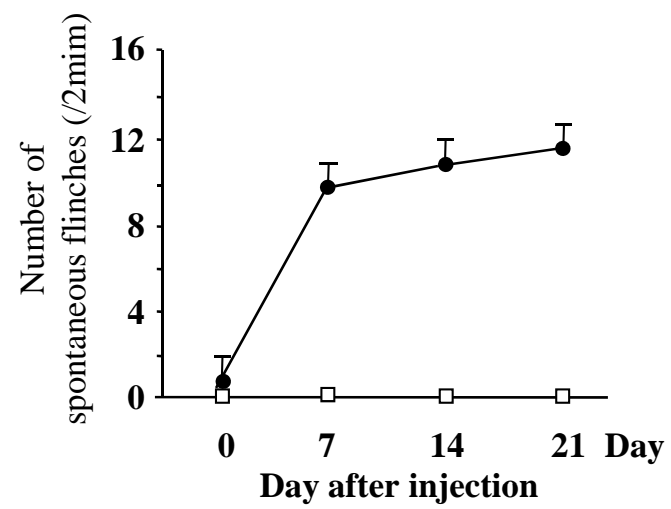

\section{B-3. Weight bearing}

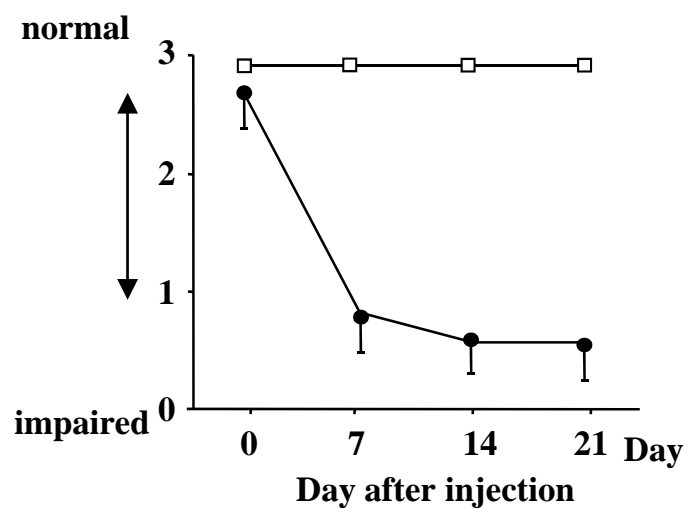

B-2. Limb use

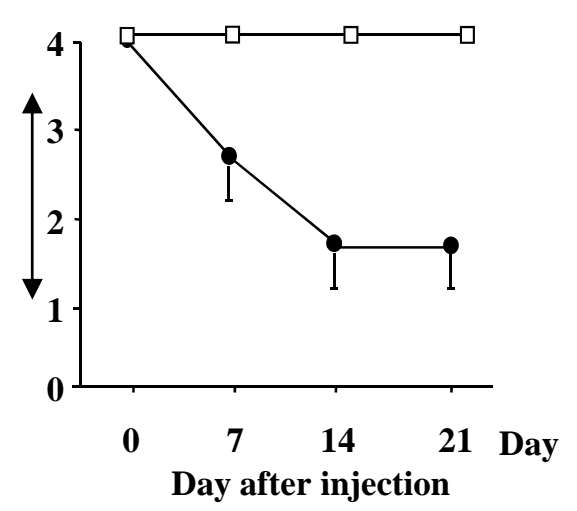


Normal condition

Primary afferent neuron

Spinal interneuren

(excitatory)

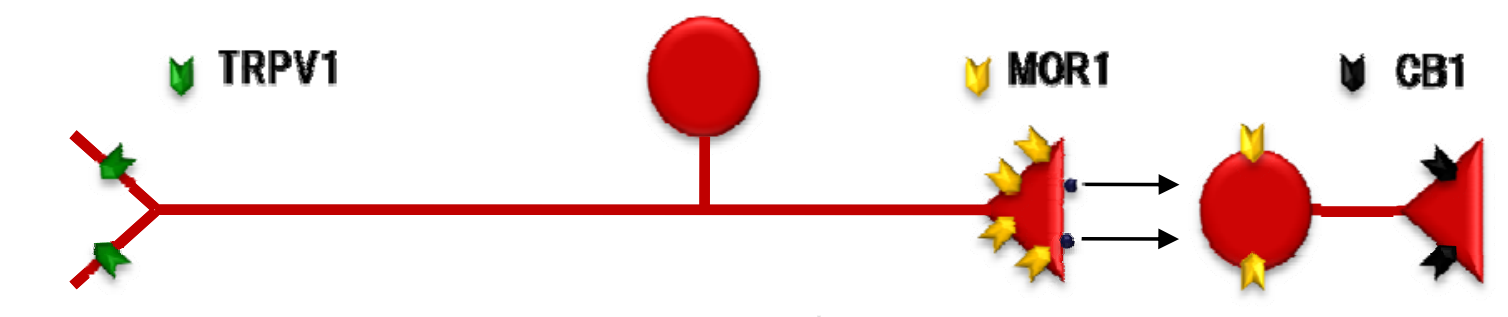

Peripheral terminal

Central terminal

Bone cancer pain

4 TRPV1 $\uparrow \uparrow$

y MOR1 $\downarrow \downarrow$

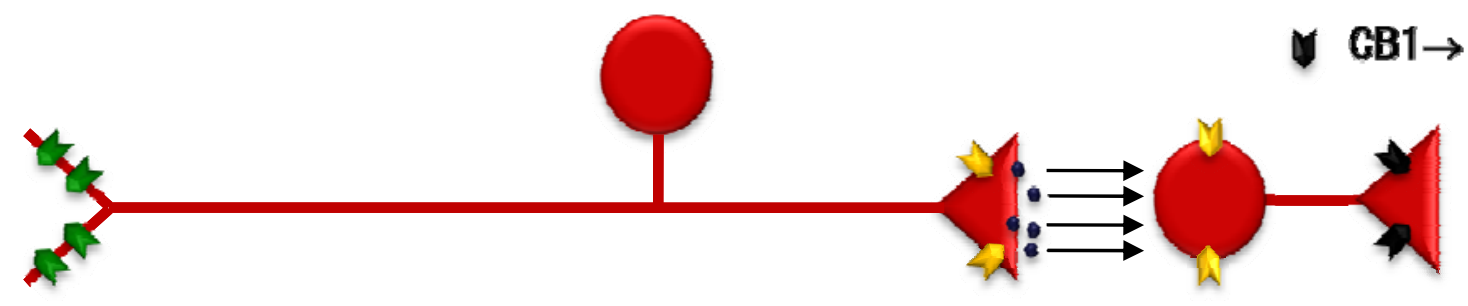

